age-matched healthy adults in judging faces on trustworthiness and gender (control task). All patients with bvFTD meet current diagnostic criteria for possible bvFTD and underwent a standardized neuropsychological assessment, a 3T MRI and a cerebrospinal fluid analysis. As stimulus material 40 unfamiliar faces were presented. Participants had to judge the trustworthiness of each face on a 7-point-Likert-scale. All faces have been created out of a sample of 1000 faces (500 male, 500 female) which were rated in respect to trustworthiness. The $10 \%$ most and the $10 \%$ least trustworthy looking male and female faces were used to create 4 averages (un)trustworthy looking male, (un)trustworthy looking female). In a second step, these faces were used as endpoints to create two 8-step trustworthiness continua by morphing procedure using Psychomorph (perceptionlab.com). Results: bvFTD patients judged untrustworthy-looking faces as trustworthy/ approachable and trustworthy-looking faces as unapproachable, but there was also a small subgroup of patients that almost behaved like normal controls. Patients had no difficulties evaluating faces on gender. Conclusions: Our findings are consistent with the idea that bvFTD affects the recognition of emotional signals like facial expressions. However, a subgroup exists which is not impaired in this subtask. Detailed Investigation of social cognition might help to better characterize patients with bvFTD.

\section{P4-160 TYPE 2 DIABETES MELLITUS: LONGITUDINAL IMPACT ON COGNITION IN PERSONS WITH NORMAL COGNITION, MCI, AND DEMENTIA}

Victoria E. Herberger ${ }^{1}$, Elise K. Hodges ${ }^{1}$, Bruno Giordani ${ }^{1}$, Jian Zhu ${ }^{1}$, Hiroko H. Dodge ${ }^{2},{ }^{1}$ University of Michigan, Ann Arbor, Michigan, United States; ${ }^{2}$ Oregon Health \& Science University, Portland, Oregon, United States and University of Michigan Health System, Plymouth, Michigan, United States. Contact e-mail: vherberg@med.umich.edu

Background: Though prior research suggests that type 2 diabetes mellitus (T2DM) is associated with an increased risk of Alzheimer's disease (AD) and Mild Cognitive Impairment (MCI), the potential contribution of T2DM to $\mathrm{AD}$ progression is unclear with mixed findings reported. Here we examine the impact of diabetes on cognition over time in individuals with normal cognition, MCI or dementia stratified by APOE4 status. Methods: Patients $(\mathrm{N}=15,460)$ from the National Alzheimer's Coordinating Centers (NACC) dataset (frozen 6/2/2013) were divided by presence of T2DM ('T2DM+' and 'T2DM-'), APOE4 status ('APOE4+' and 'APOE4-'), and NACC consensus diagnostic categories (amnestic and non-amnestic MCI, AD, vascular dementia [ $\mathrm{VaD}]$, and normal cognition). Mixed effects models controlling for age, gender and years of education and their interactions with time were included examining potential impact of T2DM on trajectories of neuropsychological test results. Participants averaged 3 visits $(\mathrm{SD}=2)$ with an average follow-up duration of 2.7 years $(\mathrm{SD}=2.2)$. Results: At baseline, the APOE4+, T2DM+ amnestic MCI and normal cognition patients showed worse performance in executive functioning $(\mathrm{p}<0.006$, multiple correction adjusted $\mathrm{p}$-value) and processing speed $(\mathrm{p}<.0001)$ compared with T2DM-. Attention span was also lower for the APOE+, T2DM+ amnestic MCI group $(\mathrm{p}<.0001)$. No significant differences were observed in the APOE4+ nonamnestic MCI, AD, or VaD groups. For the APOE4-, T2DM+ with normal cognition scored lower in executive functioning $(\mathrm{p}<.0001)$ and processing speed $(\mathrm{p}<.0001)$, in addition to learning $(\mathrm{p}=.001)$ and memory $(\mathrm{p}<.0001)$. No significant differences were observed in the APOE4- non-amnestic MCI or $\mathrm{VaD}$ groups, though the $\mathrm{AD}$ group performed worse on processing speed $(\mathrm{p}=.005)$ at baseline. In regards to longitudinal change, among APOE4$\mathrm{AD}$ group, we found significantly less decline in executive functioning $(\mathrm{p}=0.003$ ) among those with $\mathrm{T} 2 \mathrm{DM}+$, in comparison with those with T2DM-. Conclusions: In our study, the T2DM diagnosis conferred greater cognitive difficulties at baseline, regardless of APOE4 status, across amnestic $\mathrm{MCI}$ and cognitively intact older adults, but not non-amnestic MCI, AD, or $\mathrm{VaD}$ groups. The slower declines in cognitive functions observed among $\mathrm{T} 2 \mathrm{DM}+$ might suggest their reduced $\mathrm{AD}$ pathology despite the lower baseline cognitive functions, in comparison with T2DM- patients. Effects of medication will be discussed.

\section{P4-161 NEUROANATOMICAL ASSOCIATIONS WITH IMPAIRMENT IN ACTIVITIES OF DAILY LIVING IN ALZHEIMER'S DISEASE}

William Jonathan McGeown ${ }^{1}$, Michael Fraser Shanks ${ }^{2}$,

Annalena Venneri ${ }^{3},{ }^{1}$ University of Strathclyde, Glasgow, Scotland, United Kingdom; ${ }^{2}$ University of Sheffield, Sheffield, South Yorkshire, United Kingdom; ${ }^{3}$ University of Sheffield, Sheffield, South Yorkshire, United Kingdom. Contact e-mail: william.mcgeown@strath.ac.uk

Background: Performance on Activities of Daily Living (ADL) tasks can influence quality of life in patients with Alzheimer's disease (AD) and are associated with the level of care required. Further insight into the neural underpinnings of ADL deficits would be advantageous for prognosis and treatment. This study, using voxel based morphometry, investigated the brain regions which are most closely associated with impairments to independent living. Methods: T1-weighted 3D Magnetic Resonance Imaging (MRI) scans were acquired from twenty-three patients with $\mathrm{AD}$ (mean age 77.13 (SD 8.96), mean education 11.26 (SD 3.03)). The patients were in the minimal to mild stages of AD (mean MMSE score 22.78 (SD 2.89), range 18 28). Functional ability was measured by informant report (caregiver/spouse) using an extended version of the Katz ADL Scale (score range 0-32), and patients were assessed with several clinical and neuropsychological instruments. The MRI scans were segmented and normalised using the VBM8 Toolbox for SPM8. Brain size was controlled prior to statistical modelling. Grey matter (GM) images were smoothed with a $12 \mathrm{~mm}$ Gaussian filter and entered into a multiple regression model to assess the relationship between ADL score and GM (covarying for age, education and sex). Results: The mean ADL score was 22.91 (SD 4.14) (score range 12-28). Decreased functional ability was associated with lower grey matter volume within the prefrontal cortex, including the right inferior frontal gyrus, anterior cingulate gyrus and medial frontal gyrus. Scores on Neuropsychiatric Inventory and Geriatric Depression Scale correlated significantly with the ADL score. Conclusions: Atrophy within the prefrontal cortex is associated with reports of lower functional performance. The findings suggest that a drop in everyday skills might signal progression of atrophy in the frontal lobes beyond a critical threshold leading to overt behavioural changes. In parallel with reduction of functional capacity there may be implications for patient management given the association with symptoms of mood disorders and other neuropsychiatric symptoms.

\section{P4-162 SEMANTIC PROCESSING IN MILD ALZHEIMER'S PATIENTS}

Deniz Buyukgok ${ }^{1}$, Isin Baral Kulaksizoglu ${ }^{2}$, Oget Oktem Tanor ${ }^{3},{ }^{1}$ Istanbul Medicine Faculty, Istanbul, Turkey; ${ }^{2}$ Istanbul Medicine Faculty, Istanbul, Turkey; ${ }^{3}$ Istanbul Science University, Istanbul, Turkey.

Contact e-mail: deniz.buyukgok@istanbul.edu.tr

Background: It is shown that the language process abilities such as verbal fluency and confrontational naming are deteriorated in Alzheimer's disease (AD). It is noted in particular that the verbal fluency is affected in early stages whereas the confrontational naming is distorted in later stages. Our hypothesis is that the deterioration in confrontational naming ability is more related with the decline in semantic fluency than in phonemic fluency in early $\mathrm{AD}$ patient. Language is a network process, therefore it can be stated that the disruptions show themselves in every area that is connected with language. So, as a language function, categoric fluency should be affected as the same level as lexical fluency. The aim of this study is to expose the impairment pattern of language processes beginning from the early stages of the disease. Methods: Among the 143 patients who submitted to Geriatric Psychiatry Department of Istanbul Medical Faculty with subjective 